\title{
FIEBERNDE GESCHLECHTER ODER PRZYBYSZEWSKIS SUCHE NACH EINER LITERARISCHEN SPRACHE ÜBER DAS BEGEHREN
}

\begin{abstract}
Feverish sexualities. Przybyszewski in search of a literary device for sexual desire
The main thesis of the article is that Przybyszewski allows his protagonists to suffer by elevated temperature or fever to defend them (and their acts) from moral judgement. On the basis of this strategy he becomes free to work out his literary experiments with the aim of finding an adequate language for the description of sexual desire.
\end{abstract}

KEYwORDS: Sensuality, Przybyszewski, Narrative Strategie, Body, Sex, Mind, Soul, Desire

Stanisław Przybyszewski studierte zwar Medizin, aber für Temperatur, Fieber und kranke Zustände interessierte er sich nicht als Arzt, sondern als Schriftsteller. Viele seiner Protagonisten fiebern und bekommen bewusstseinsverändernde Zustände. Medizinisches Vokabular nutzt Przybyszewski jedoch für deren Beschreibungen; es dient ihm für poetische Bilder, in denen organische Prozesse und psychische $\mathrm{Zu}$ stände dargestellt werden, wie etwa in der Totenmesse von 1893. Hier beschäftigen ihn insbesondere Neurosen und Psychosen, in welchen er „die fortschreitende Entwicklung des menschlichen Geistes“" zu sehen glaubt, ${ }^{1}$ denn in ihnen, schreibt er,

[...] liegen die Samenkeime eines neuen, bis jetzt noch nicht klassifizierten Empfindens; sie sind es, in denen das Dunkle sich mit der Morgenröte des Bewußtseins rötet und die unterirdischen Riffe sich über das Niveau der Meeresfläche heben. ${ }^{2}$

\footnotetext{
${ }^{1}$ Stanisław Przybyszewski: Totenmesse. In: ders.: Werke, Aufzeichnungen, Briefe. Paderborn 1990, Bd. I, S. 7-41, hier S. 9.

${ }^{2}$ Ebd.
} 
Als ehemaliger Medizinstudent wusste Przybyszewski, dass psychische Krankheiten ohne Fieber verlaufen. Ihm geht es jedoch nicht um deren klinisches Bild, sondern um die Thematisierung dessen, was im Zustand der Unberechenbarkeit, des Kontrollverlustes zutage tritt. Darauf verweist er in der Einleitung zur Totenmesse, wenn er feststellt: An einem Menschen, der an „Schreckenspsychose leidet, kann man tiefere und feinere Rückschlüsse auf die Psychologie der Zeit, auf die Natur einer wirklich individuellen Veranlagung gewinnen“" ${ }^{3}$

Gabriela Matuszek betont, Przybyszewski bleibe in seinen frühen Essays vor allem der Psychophysiologie des 19. Jh.s. verhaftet, weil er sich auf die Entwicklung des menschlichen Geistes in Anbindung an die organische Seite des Menschen, vor allem an sein Nervensystem, konzentriere. ${ }^{4}$ Dies mag wohl für dessen Essays über Hansson, Chopin und Nietzsche zutreffen. Wenn man sich aber seine literarischen Gestalten näher anschaut, so fällt auf, dass es auch um deren sexuelles Begehren geht; in den Augenblicken, in denen es aufkommt, steigt ihre Temperatur. Aber krankhaft zu fiebern beginnen sie, sobald sie in ethisch-moralische Konflikte geraten, Konflikte zwischen ihren sexuellen Wünschen und gesellschaftlichen Normen, etwa dem Ideal der Treue zur geliebten Person in Geschlechterverhältnissen.

Die thematische Zusammenführung von Geschlechterbeziehungen und krankhaften, fiebrigen Zuständen scheint eine besondere Strategie Przybyszewskis im literarischen Umgang mit Sexualität zu sein. Jens Malte Fischer siedelt ihn zwischen Autoren wie Leopold von Sacher-Masoch, Joris-Karl Huysmans und Gabriele D'Annunzio an, die das Thema der Sexualität in der zweiten Hälfte des 19. Jh.s. auszuloten suchten. ${ }^{5}$ Przybyszewskis nekrophile Liebesszene in Totenmesse hält er für eine originelle Übersteigerung dessen, was um die Jahrhundertwende zum Thema ,dekadenter Sexualität" geschrieben wurde. ${ }^{6}$ In ihr imaginiert ein fiebernder IchErzähler eine sexuelle Annäherung mit einer weiblichen Leiche. Vor der Darstellung dieser Imagination entwickelt Przybyszewski rhapsodische Ausführungen über die weltkonstituierende Kraft des Geschlechts, aus dem Seele und Geist entstehen, die das Geschlecht als ihren Ursprung verdrängen. In Homo Sapiens, einer Trilogie, die 1895-1896 entstand, versucht Przybyszewski die Gewichtigkeit der sexuellen Wünsche und Erfahrungen für individuelle Existenz eines Menschen zu beschreiben. Dabei geht es ihm um entsprechende Sprache, in der er die Intensität des sexuellen Begehrens einem Publikum vermitteln kann, für welches Sexualität immer noch stark tabubehaftet war. Im Zusammenhang mit der radikalen Ausgangsthese: „Am

\footnotetext{
${ }^{3}$ Ebd., S. 10.

${ }^{4}$ Vgl. Gabriela Matuszek: Między pustkq transcendencji a szaleństwem zmysłów. O wczesnej eseistyce Stanistawa Przybyszewskiego [Zwischen der Leere der Transzendenz und dem Sinnenrausch. Die frühe Essayistik von Stanisław Przybyszewski]. Einleitung zu: Stanisław Przybyszewski: Synagoga Szatana i inne eseje. Kraków 1997.

${ }^{5}$ Jens Malte Fischer: Fin de siècle. Kommentare zu einer Epoche. München 1978, S. 58f.

${ }^{6}$ Ebd., S. 60 und 220.
} 
Anfang war das Geschlecht" ", und mit deren Auslegung steht das Begehren im Zentrum dieser Trilogie sowie seines ganzen Schaffens. Während er das Begehren in der Totenmesse als ein Phänomen metaphorisch erfasst, betrachtet er es in Homo Sapiens als einen Teil der conditio humana analytisch, indem er es mit der Lebenspraxis eines Protagonisten, Erik Falk, in unterschiedlichen Beziehungskonstellationen konfrontiert. Insgesamt bemüht sich Przybyszewski, der wie Fischer bemerkt, ,ein Unikat unter den deutschen Autoren des Fin de siècle“ war, er habe „eigenständig und als einziger [...] Aspekte der französischen Décadence" weiterverarbeitet ${ }^{8}$ - als der geniale Pole und Freund Richard Dehmels im Kreis der Berliner Boheme um die Salonfähigkeit des Themas des sexuellen Begehrens in der Literatur. Er war hierbei weder am medizinischen Geschlechterdiskurs noch am Pathos der literarischen Liebesdarstellung interessiert. Um nicht Gefahr zu laufen, mit seinen Texten in die verpönte Ecke der pornographischen Literatur gedrängt zu werden, vermied er gekonnt die direkte Schilderung des sexuellen Verkehrs. In ihrem Buch Godzina cudu. Miłość i erotyzm w twórczości Stanisława Przybyszewskiego [Stunde des Wunders. Liebe und Erotik im Schaffen von Stanisław Przybyszewski] beschreibt Katarzyna Badowska mehrere diesbezügliche Vermeidungsstrategien dieses Autors, vor allem die Aneinanderreihungen von entsprechenden Metaphern, Verben und Adjektiven, mit denen er die Annäherung der Begehrenden zueinander dynamisiert und eine besondere erotische Spannung zwischen ihnen erzeugt. ${ }^{9}$ Den Wortschatz aus dem semantischen Umfeld der Sexualiät entwickelt Przybyszewski durch die Anhäufung von stilistischen Mitteln fast so, wie es Michel Foucault viele Jahrzehnte später in seiner Geschichte der Sexualität für die Zeiten beschreibt, in denen die Sexualität besonders stark tabuisiert war. Begriffe aus der Medizin dienen Przybyszewski nicht dazu, Aussagen zu neutralisieren, sondern vielmehr zum Aufbau eines Kontrastes zwischen der Neutralität und Intensität der Beschreibung von körperlichen Empfindungen und seelischen Qualen. ${ }^{10}$ Seine Liebesszenen werden dadurch paradoxerweise körperlos, denn er schreibt zwar über Empfindungen und deren Omnipräsenz, aber markiert sie mit neurasthenischen Zuständen und vor allem mit unterschiedlichen Temperaturen und Fieber. Manchmal entsteht der Eindruck, dass sinnliche Empfindungen des Individuums nur in fieberhaften Zuständen möglich sind. Nur in ihnen erfüllen sich innige Wünsche, ${ }^{11}$ zu denen ein Mensch ohne Fieber, der nor-

\footnotetext{
${ }^{7}$ Przybyszewski: Totenmesse, S. 10.

${ }^{8}$ Fischer: Fin de siècle, S. 231.

${ }^{9}$ Vgl. Katarzyna Badowska: Godzina cudu. Mitość i erotyzm w twórczości Stanistawa Przybyszewskiego. Łódź 2011, S. 45ff.

${ }^{10}$ Ulrich Steltner schreibt sogar, dass etliche Nomina bei Przybyszewski aktive und passive Handlungsträger sind. Vgl. Ulrich Steltner: Stanistaw Przybyszewski - Schriftsteller in zwei Nationalliteraturen [online], <http://www.slawistik.uni-jena.de/tl_files/tl_files/Institutsverlag/Kollar/11steltner.pdf> [12.12.2015].

${ }^{11}$ Um 1900 gab es in der deutschen Literatur mehrere Versuche, mit Normüberschreitung zu experimentieren. Auf diesem Weg versuchte u. a. Otto Gross seinen Patienten und Patientinnen zu neuer
} 
mentreu zu leben sucht, sich nicht bekennen würde. Sogar der fiebernde Kranke, wie Przybyszewski zeigt, der sich dieser Wünsche in hellen Momenten bewusst wird, spürt seine Ratio, die nicht zulässt, dass er ihnen mit Überzeugung problemlos nachgeht. Ihn überfallen Zweifel, Gewissensbisse und der Zustand verschlimmert sich weiter.

So begehrt Erik Falk im ersten Band der Trilogie Homo Sapiens mit dem Titel Über Bord Isa, die eigentlich seinen Freund heiraten sollte, sich aber im letzten Moment anders entscheidet und bei Falk bleibt. Ähnliches erlebt er, als er im zweiten Band (Unterwegs) die junge Marit verführt und diese Selbstmord begeht, nachdem sie erfahren hat, dass er verheiratet ist und seine eigene Frau liebt. Auch auf Janina machen seine Anziehungskraft und sein Begehren einen großen Eindruck und sie verliebt sich in ihn. Im dritten Band (Im Malstrom) bekommt sie ein Kind von ihm. Sie bräuchte seine Hilfe, die er aber nicht leisten will, aus Angst, seine Ehe zu gefährden.

Das Begehren bestimmt Falks Handeln als eine Kraft, die im Menschen schlummert und in einer unklaren Form sein Leben, seine Verhaltensweise, sein Empfinden unkontrollierbar beherrscht. Dieser Mechanismus erinnert zwar an den, welchen Sigmund Freud einige Jahre später in seinen Vorlesungen zur Einführung in die Psychoanalyse als Verdrängung definiert. Przybyszewski interessiert jedoch dieser Mechanismus nicht, sondern vor allem jene Momente, in denen die Verdrängung nicht funktioniert. Deswegen entwickelt er in der Totenmesse eine poetische Vision, in der es zu einer Dominanz der Seele und des Geistes über das Geschlecht kommt. Die verheerende Rolle dieser konfliktträchtigen Triade Geschlecht-SeeleGeist zeigt Przybyszewski - wie schon erwähnt - exemplarisch an den Qualen eines Ich-Erzählers, der sich dem Drang der Triebe nicht widersetzen kann und beim Liebesspiel mit einer weiblichen Leiche in einen Rauschzustand fällt. Er versucht anfänglich, sich gegen die Anziehungskraft der Leiche zu wehren, aber das schafft er nicht. Er fühlt sich von ihr sexuell so stark angezogen, dass er sich ihr körperlich nähert. Am Ende stößt sie ihn gegen die Brust und er fällt müde zurück, wie nach einem vollzogenen Geschlechtsakt:

Mich überkam ein geschäftiges Treiben. Ich hob das Lid mit kunstgerechtem Griffe langsam hoch, geschäftsmäßig, wie bei der Augeninspektion; aber meine Finger glitten das Gesicht herab, sie betasteten es, ein Fieberparoxysmus überkam mich, ich arbeitete mit autonomen Gliedern, ich hatte die Empfindung, daß mein Kopf mir durch das Fenster flöge, und ich lachte und schrie und fühlte meine eignen Laute auf mich zurückprallen, wie Steinwürfe, - ich küßte ihr Gesicht, ich riß und sog an ihr, und plötzlich biß ich mich mit geifernden Lippen, wie ein Vampir, schrill in ihre Brust hinein. ${ }^{12}$

Selbsterkenntnis zu verhelfen. Damit der Zwang der Anpassung an Normen bei ihnen nachlässt, verabreichte er ihnen während der psychoanalytischen Therapie Rauschmittel.

${ }^{12}$ Przybyszewski: Totenmesse, S. 30. 
Dies geschieht in einem Fieberparoxysmus, nachdem er der Leiche gesagt hat: „Siehst du, wenn man degeneriert ist und krank, dann weiß man niemals um seine Zustände; sie verändern sich nämlich fortwährend, jetzt noch Liebe und Glück und im selben Augenblick $\mathrm{Haß}$ und Ekel“. ${ }^{13}$ Seinen krankhaften Zustand verfolgt er Schritt für Schritt, spürt, dass sein Ich dabei zerfällt. Ihn verfolgen selbstzerstörerische Gedanken, zugleich aber kämpft er gegen den Wunsch, Selbstmord zu begehen. Er fühlt sich einsam, kann aber keinen Menschen leiden, liebt nur ein objekthaftes Du. Diese Liebe verwandelt sich im Geschlechtsakt dann schlagartig in ein brutales, rücksichtsloses Begehren:

Und so mußte ich deine Formen, deine Bewegungen lieben, so mußte mich die Stimmung, die du strömtest, berauschen, weil mein Geschlecht mir meine Seele auf dich eingerichtet hatte, dass sie dich zum Fraße ihm zuführe, zum Molochopfer überliefere. ${ }^{14}$

Diese Liebesbeziehung gehört zwar der Vergangenheit an, sein Liebesobjekt scheint jedoch jetzt jene Tote zu sein. Das Ich sehnt sich nach seinen Liebeserlebnissen so sehr, dass sein Begehren ihn im fieberhaften Zustand in die Arme der Leiche treibt:

Ich sehnte mich in heißem Fieber nach dem Werdeprozeß, wie die Formen meines Geistes sich in deinen Körper kleideten, die Zuckungen meiner Muskeln dich ins Leben leiteten, wie du nach dem Muster meines Geistes wurdest und ein neues Wesen begann. ${ }^{15}$

Das Du wird im Liebesakt vom liebenden Ich beeinflusst, die Geliebte dient dem Ich zur Selbstsuche, die hoffnungslos ist, weil dieses Selbst in diesem Erlebnis verschwindet. Das fiebernde Ich bekennt:

Das Mysterium der Nacht und des Abgrunds - in Dir wollte ich es sehen. Ich griff nach ihm mit fiebernden, qualstöhnenden Fingern; wie feine Lanzettenspitzen grub ich sie in deine Tiefe, und immer entschlüpfte es mir, glitt tiefer, verschwand. ${ }^{16}$

Das kranke Ich spürt in sich die quälende Kluft, die durch das geheimnisvoll wirkende Geschlecht der Seele und dem Geist zum Trotz entstanden ist. Erst die orgiastische Begegnung mit der Leiche ermöglicht ihm, sich selbst wieder wenigstens ansatzweise zu spüren:

Mit der zuckenden, schauernden, hirnzerrüttenden Leidenschaft, mit der fiebernden Glut, die mein Gehirn durchtobt, mit der ungestümen Kraft meiner lusterstarkten Glieder will ich mich von dem Erdbeben deines Fleisches schütteln lassen, nichts fühlen als die bleiche Hitze Deiner Glieder, nichts hören als das jagende Sausen meines Blutes, nichts empfinden als das stechende, brutale Weh des Liebesdeliriums, - ich will aufhören zu leiden in dem Siegesdithyrambus des Geschlechtes, der tosenden Brandung einer schauerlichen Symphonie des Fleisches. ${ }^{17}$

\footnotetext{
${ }^{13}$ Ebd., S. 28.

${ }^{14}$ Ebd., S. 18.

${ }^{15}$ Ebd.

${ }^{16}$ Ebd.

${ }^{17}$ Ebd., S. 23.
} 
Um dieses Gefühl der „fiebernden Glut“ weiter zu intensivieren, verlangt er noch: „brenn' es mir in meine Glieder, senge es auf meine Lippen, atme es in mich hinein, dies heiße, gierige ekstatische: Ich liebe dich!“‘18

Die erhöhte Temperatur erscheint in der Liebesekstase des Ichs auf mehreren Ebenen: Seine Finger sind fiebernd, seine Leidenschaft ist fiebernde Glut und das der Frau abverlangte Liebesbekenntnis ist heiß. Diese Empfindung der Hitze erlebt er wie beim Musikhören: Es „schmerzt ihn die Glut“, ihre Klänge, sie „stachen wie dünne, bis zur Weißglut erhitzte Nadeln in mein Gehirn hinein“. ${ }^{19}$ Als der Zustand des Deliriums, in dem die oben angeführten Empfindungen und Visionen aufkommen, kurz nachlässt, fühlt das Ich zuerst den „kalten Schweiß“, hat „ein eisiges Gefühl“, „Kältegefühl“ und heiße Tränen: „Namenloses Mitleid überkam mich; heiße, große Tränen rollten über meine Wangen, und mir schien, dass an meinen Beinen etwas niederlaufe - mich fröstelte wohl“ ${ }^{20}$ Die Empfindung der Kälte nimmt zu, sobald er wieder zu Bewusstsein kommt.

Die Totenmesse endet mit dem geheimnisvollen Satz: „Die rückschreitende Metamorphose kann beginnen...“. ${ }^{21}$ Wenn man ihn auf den Anfang des Textes bezieht, kann man diese rückschreitende Metamorphose als Weg zur Aufhebung der Abspaltung der Seele und des Geistes von dem Geschlecht im Menschen verstehen.

Auf solch einen Weg scheint Przybyszewski die Protagonisten seiner Romane immer wieder zu schicken. Der Titel seiner Trilogie Homo Sapiens zielt auf eine Verallgemeinerung hin, als gehöre der Drang zu solch einer Metamorphose zur conditio humana. So wird in jedem der drei Bände eine Geschichte von Erik Falk erzählt, in der das Begehren die Oberhand gewinnt. Obwohl er einen hohen Preis dafür zahlt, kann er sich diesem Begehren nicht widersetzen. Er erkennt bei sich: „Ja, nun wußte ich bestimmt: es ist das Geschlecht. Und nun wuchs es in mir und wuchs unaufhörlich und ließ mir keine Ruhe, und ich muß: ja ich muß! Warum? Ich weiß es nicht“ ${ }^{22}$ Indem er Beziehungen mit Isa, Marit und Janina eingeht, verliert er im ersten Fall seinen Freund, im zweiten trägt er zum Selbstmord von Marit bei, und letztendlich verliert er seine geliebte Ehefrau, als sie von diesem Tod erfährt und auch davon, dass er mit Janina ein Kind hat.

Alles in allem sind das banale Liebesgeschichten, wodurch diese Trilogie in der Forschung nicht besonders hoch geschätzt und meistens als ein stark autobiographischer Text abgewertet wird. Es sind aber Przybyszewskis erste Romane, in denen sein Konzept von Geschlechtlichkeit und Sinnlichkeit nicht mehr rhapsodisch, also in Form von losen assoziativen Bildern formuliert, sondern in lebenspraktischen

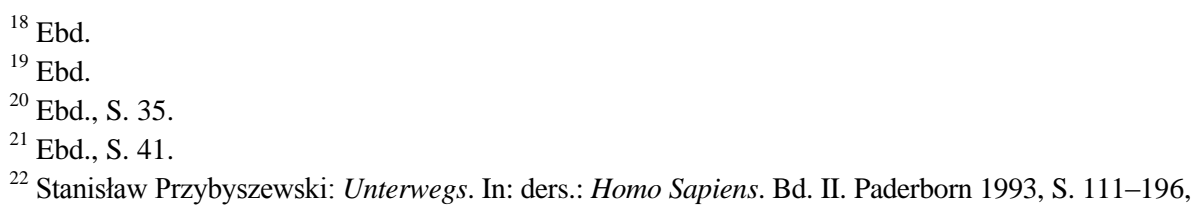


Konstellationen eines Individuums durchgespielt wird. Es sind unterschiedliche Liebesbeziehungen, die sein Falk eingeht: Sie folgen nicht nur aufeinander, sondern manche verlaufen parallel zueinander und sind miteinander verflochten. Wenn Falk sich wehrt, Isa nicht begehren will, um seine Freundschaft zu Mikita, die mit dessen warmem Handdruck bezeugt wird, nicht zu gefährden, führt es ihn zu Janina, die seine Zuneigung mit Dankbarkeit annimmt. Sobald Falk von seiner Frau wegen des Unglücks von Janina und Marit, das er verursacht hat, verlassen wird, intensiviert er seine Beziehung zu Olga. Diese Verflechtungen suggerieren, dass das sexuelle Begehren ein Aufbegehren gegen jedes Verbot sei, als eine Kraft, die jede Norm als Produkt der Vernunft torpedieren müsse. Der Mensch erscheint bei Przybyszewski als ein Medium, in welchem sich immer wieder das abspielt, was er in der Totenmesse beschreibt - die Kluft zwischen dem Geschlecht und dessen Gebilden, die sich von ihm abgespalten haben: der Seele und dem Geist. Durch die unbeherrschte Wirkung des Geschlechts kommt es zur Selbstentfremdung des Ichs, das über seinen Willen nicht frei verfügen kann.

Falks Körper reagiert im ersten Band Über Bord trotz aller Bemühungen um Selbstdisziplin in einer unbeherrschten Weise auf Isa: „Es war ihm, als ströme ihm unaufhörlich eine Hitze in die Augen, ja, es war als sauge sein Körper ihre ... ihre Wärme in sich ein .... ${ }^{63}$ Er versucht es zu verstehen und überlegt: „Wie kam es, daß in einer Stunde ein Weib zu ihm in Beziehung trat, in sein Gehirn übertrat als eine Art Fremdkörper, um den sich nun sein Denken, sein ganzes Fühlen sammelte, in den sein Blut goß ..." ${ }^{24}$ Noch ehe er eine Antwort finden kann, weicht er Isa aus und geht zu Janina, zu der „,vor Jahren noch ,Brand“ in seinem Kopfe herumspuckte“. 25 Dieser Prozess, dem Falk unterliegt, ist nicht geschlechtsgebunden, denn Isa als Frau spürt auch eine „weiche, angenehme Empfindung der Wärme“, als sie sich an einen Tanzabend mit Falk erinnert. ${ }^{26}$ Janina wiederum will seine Nähe, unabhängig davon, was mit ihm und seinen anderen Frauen geschieht. Marit glaubt ihm jedes Wort, obwohl sie genau spürt, dass er lügt. „Sie konnte nicht denken, sie fühlte nur den wilden, begehrlichen Schauer durch ihren Körper zucken.“27

Wenn man die Handlung dieses Romans, wie auch der ganzen Trilogie, entlang der sich verändernden Temperaturen verfolgt, erkennt man, in welcher psychischen Phase sich der Protagonist befindet. Er fiebert aus Begehren, er fiebert und taumelt, wenn er sich in einer Konfliktsituation befindet, und er fiebert so sehr, dass er sich hinlegen muss oder bewusstlos wird, sobald er erkennt, dass sein Begehren ihn in eine ausweglose Situation getrieben hat.

\footnotetext{
${ }^{23}$ Stanisław Przybyszewski: Über Bord. In: ders.: Homo Sapiens. Bd. I. Paderborn 1993, S. 13-109, hier S. 27.

${ }^{24}$ Ebd., S. 37.

${ }^{25} \mathrm{Ebd}$.

${ }^{26}$ Ebd., S. 55.

${ }^{27}$ Przybyszewski: Unterwegs, S. 148
} 
Im ersten Band will er sowohl Mikitas Freundschaft als auch Isas Liebe. Er begehrt dieselbe Frau wie dieser, und fühlt gleichzeitig, dass er sich in diesem Begehren rückhaltlos verliert: „War Liebe nur eine Krankheit, ein Fieber, um faulende Stoffe auszuscheiden, - ein Genesungsprozess - ein Blödsinn - ein - ein - Herrgott! Wie der Zug raste. ${ }^{28}$ Die Flucht vor diesem Konflikt zur Mutter gelingt nicht. Er kehrt um, und sein Zustand verschlechtert sich radikal: „Seine Glieder schmerzten ihn, aber er hatte deutlich ein Gefühl, dass er seine Krankheit überwunden hat.“'“29 Diese Linderung ist jedoch nur von kurzer Dauer, denn „dann kam der Strudel, der Wirbel ... Das Gehirn fing an, sich um sich selbst zu drehen, und kreiste schneller und schneller in den abgründigen Trichter des Geschlechtes hinab ... ${ }^{* 30}$ In einem Traum will er Mikita über Bord werfen. Sobald er zu sich kommt, friert er. Auch Mikita leidet: „Es fing an in seinem Gehirn zu fiebern“, ${ }^{31}$ weil er nicht nur seine Geliebte, sondern auch seinen Freund verliert. Er hält die Spannung nicht aus und begeht Selbstmord. Falk wehrt sich gegen Gewissensbisse, die in ihm nach dem Tod aufkommen. Auf der Reise nach Paris mit Isa sagte er sich: „Das Alles mußte so geschehen. Sonderbar, dass er immer wieder in die alten Vorstellungen vom freien Willen verfiel, von einem Willen, der handeln kann ... ${ }^{32}$ Und das ist Falks Haupterkenntnis: Den freien Willen gibt es nicht, weil das Geschlecht unbeherrschbar ist und nach eigenen Gesetzen über das menschliche Handeln verfügt.

Im zweiten Band, Unterwegs, sucht Falk schon nach keiner Rechtfertigung seiner Handlung, sondern leidet darunter, was er der jungen Marit antut und wogegen er sich aber nicht wehren kann. Er fiebert nach ihr und belügt sie, um sie zu erobern. Danach wird er eine Woche lang krank, denn er spürt, dass Marit es nicht überleben wird. Als er zu Bewusstsein kommt, erblickt er seine Frau am Bett, und ist beruhigt. Ein Gedanke geht ihm nur noch durch den Kopf - „Marit ist wohl tot“ - und er schläft wieder ein. Nach den vielen Hitzewallungen der Werbung um Marit und den ursprünglichen Qualen wegen des Betrugs, der sie in den Selbstmord treibt, folgt eine Genesung, mit der der nächste Zyklus aus Begehren, Befriedigung und Reue endet, bis ein neuer unweigerlich beginnt. Der dritte Band Im Malstrom zeigt die negativen Folgen dieser Zyklen. Falk sieht ein, dass er sie nicht vermeiden kann und gerät wieder in „Verzweiflungsfieber“ ${ }^{33}$ Er bettelt die Natur um Gnade: „Liebe Natur, versuch deine boshaften, tückischen Kunstgriffe an anderen Menschen; ich kenne sie zu gut deine Kniffe und Schliche - nein! Mich zu quälen, gelingt dir nie - nie! ${ }^{34}$

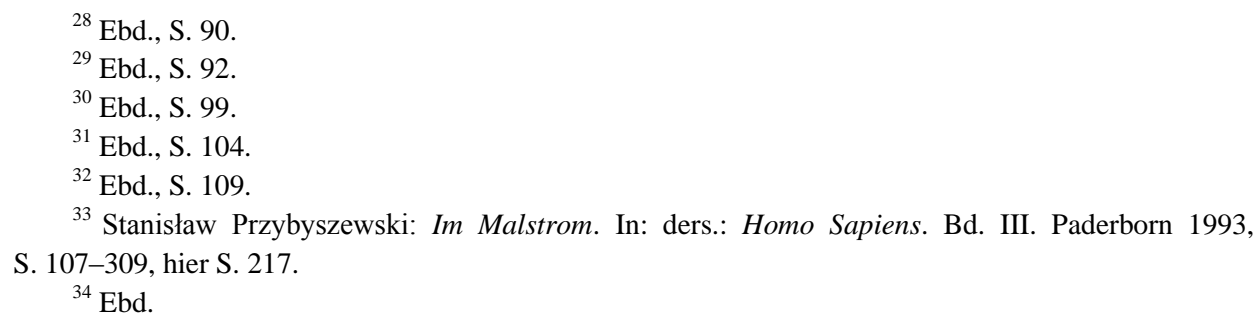

${ }^{33}$ Stanisław Przybyszewski: Im Malstrom. In: ders.: Homo Sapiens. Bd. III. Paderborn 1993, S. 107-309, hier S. 217.

${ }^{34}$ Ebd. 
Seine geliebte, jedoch schwer enttäuschte Frau verlässt ihn hasserfüllt: „Er ließ sie los. In einem $\mathrm{Nu}$ fühlte er sein Inneres zu Eis gefrieren“ ${ }^{35}$ Aber er geht nicht zugrunde. Olga ist die nächste Frau an seiner Seite, der er seine Liebe bekennt, was darauf hindeutet, dass die Wiederkehr des immer Gleichen sich vom Neuen abspielen kann.

Den Hauptteil jedes Bandes der Trilogie bildet so etwas wie ein Gezerre im Spinnennetz der Triade Geschlecht-Geist-Seele, bei dem es nur Verlierer und Verliererinnen gibt $^{36}$ - ein Thema, das für Przybyszewski nicht nur literarisch, sondern auch existenziell wichtig war. In jedem nächsten Text, was schon in Satans Kinder von 1897 und noch deutlicher in der Erzählung Synagoge des Satans aus demselben Jahr sowie in Androgyne von 1900 zu beobachten ist, wird es von ihm immer radikaler behandelt und in Anlehnung an Huysmans, den er intensiv rezipierte, mit dem Okkultismus in Verbindung gesetzt. In diesen Schilderungen dominiert die Stimmung einer unausweichlichen Qual, die Przybyszewski in die conditio humana des homo sapiens einschreibt. Dabei bedient er sich eines klassischen heterosexuellen Liebes- und Begehrensmodels: Liebe ist bei Przybyszewski eine monogame Beziehung mit Besitzansprüchen. Gegen sie wehrt sich Falk. Nach jeder sexuellen Eroberung versucht er, seine Opfer davon zu überzeugen, „daß Liebe überwunden werden muß, daß sie ein rudimentäres Gefühl sei, eine Art pathologischer Ausschlag im Geistesleben des modernen Menschen“, ${ }^{37}$ und dass sie dem Begehren im Wege steht. Deswegen ärgert sich Falk, sobald er sich selbst verliebt. Er weiß, dass er die Ansprüche der geliebten Person nicht erfüllen kann, und er will nicht das Leiden der Geliebten sehen, sobald sie erfahren, dass er in ihren Augen „kein guter Mensch“ ist.

\begin{abstract}
Aber mein Fieber ist kein physisches; ich kann logisch sprechen, und ein Mann, der Fieber hat, der kann es nicht. Nicht wahr! Also sehen Sie, Sie werden mich von den Menschen erlösen, die mich lieben. Und jeder Mensch, der mich liebt, ist mein Feind. Die Menschen, die mich lieben, quälen mich so entsetzlich. Ich muß lügen, beständig lügen, um nicht die Qual der Enttäuschung bei ihnen zu sehen. ${ }^{38}$
\end{abstract}

Früher oder später erweist sich nämlich, dass die Liebe dieser Menschen, dadurch dass er andere begehrt, von ihnen nicht als Liebe, sondern als bloßes Begehren verstanden wird. Bezeichnenderweise verwehrt Przybyszewski Falk die Einsicht, dass die Frauen Ähnliches erleben. Sie erscheinen in den Romanen und in Falks Augen immer als verbotene Frucht, die begehrt wird, als ob Begehren ohne Verbot nicht möglich wäre. Man kann sich des Eindrucks nicht erwehren, dass Przybyszewski die Grenzsituationen anders literarisch nicht kreieren kann, wenn er immer wieder eine gezielt unerträgliche Spannung in der Handlung zu entwickeln beginnt. Daher ver-

\footnotetext{
${ }^{35}$ Ebd., S. 304.

${ }^{36}$ Wie auch in Stanisław Przybyszewskis späterem Roman Satans Kinder.

${ }^{37}$ Przybyszewski: Über Bord, S. 17.

${ }^{38}$ Przybyszewski: Im Malstrom, S. 243.
} 
bindet er in der Trilogie, wie auch in dem späteren Roman Satans Kinder, das Motiv der Liebe mit einem erotischen bzw. sexuellen Erlebnis im Kontext der „verbotenen Frucht". In ihrem Rahmen wird ein traditionelles Ideal der Liebe vorausgesetzt, unter dem eine innere Verpflichtung dem Partner bzw. der Partnerin zum monogamen Zusammensein ohne Beachtung dessen, was im Leben der oder des Anderem geschieht, zu verstehen ist. Auf den ersten Blick scheint in dieser Auffassung der Liebe die Quelle der erwähnten Spannung und der Machtlosigkeit zu liegen, die den Autor Przybyszewski quält, sobald er einen Versuch unternimmt, Begehren als Grundlage der Konstitution des Menschen zu setzen. In diesem Begehren sieht er den Motor einer jeden Aktivität, aber keinen Eigenwert an und für sich. Das Begehren versucht Falk nur einmal in einer Auseinandersetzung mit dem Revolutionär Czerski aufzuwerten, indem er einen Vergleich zwischen dem politischen Mord und seinem Vergehen gegen die Frauen zieht:

[...] aber sagen Sie nur, worin sich dann Ihr Verbrechen von dem meinigen unterscheiden wird? Dadurch, daß mein Verbrechen straflos bleibt, und Sie mit dem Tode bestraft werden. Aber ich habe die Qual, und Sie haben das Glück des Opfers, ja - des Opfers [...]. ${ }^{39}$

Er beweist Czerski, dass dessen politisches Engagement bei genauerer Betrachtung seinen Ursprung im Begehren hat. Er könne nicht gesättigt werden, weil seine Frau Geliebte eines anderen geworden war, als er im Gefängnis saß. Falks Argumentation ist jedoch ambivalent. Zum Teil rührt sie aus seiner Überzeugung, zum Teil aus der Angst, dass Czerski ihn bei seiner Frau wegen Janina denunzieren könnte. Sobald die Gefahr nicht mehr bestand, war es ihm, ,als wäre das Fieber von ihm plötzlich gewichen". 40

Przybyszewski lag tatsächlich nicht daran, aus seinem Begehrenskonzept ein Erklärungsmodel für alles zu machen oder eine Alternative zur monogamen Liebe zu schaffen. Wichtiger war ihm das, was er schon zu Beginn seines Schaffens an der Novellensammlung Die Parias von Ola Hansson 1892 bewunderte:

Es bewegt sich alles auf der Grenze, wo der Schmerz schon aufgehört hatte, Schmerz zu sein und in Nirwana umgekippt ist, ein lang gedehntes, monotones, beschauliches Oum, halb wollüstiges Schauern, halb grauende Vertiefung, Hinabgleiten, Versinken, Auflösen, Auseinanderfallen. ${ }^{41}$

Diese Grenzsituation interessiert ihn - wie gesagt - mehr als die Frage, wie man jenes Hinabgleiten und Auseinanderfallen überwinden könnte. Gerade diese negativen Zustände nutzt Przybyszewski thematisch dazu, seine Beschreibungen dessen, wie seine Protagonisten sich selbst erfahren und erleben, eindrucksvoll stilistisch zu

\footnotetext{
${ }^{39}$ Ebd., S. 248.

${ }^{40}$ Ebd., S. 249.

${ }^{41}$ Stanisław Przybyszewski: Zur Psychologie des Individuums. I. Chopin und Nietzsche [online], <https://wolnelektury.pl/katalog/lektura/przybyszewski-zur-psychologie-des-individuums.html> [15.12. 2015], Absatz 180.
} 
intensivieren. Man könnte sagen, dass er ethische Normen und das Leiden, das sich mit deren Überschreitung verbindet, als einen allgemein verständlichen Rahmen literarischer Konvention und der gesellschaftlich anerkannten Umgangsformen in Geschlechterverhältnissen nutzt, um sprachliche und stilistische Möglichkeiten textueller Kreationen sinnlicher Erlebnisse zu erkunden. So nähert er sich in seinen Texten dem Tabu der Sexualität, ohne sein Publikum gänzlich zu schockieren. In der Trilogie verfährt er damit sehr behutsam, indem er seinen mutigen Bildern immer eine Korrektur hinzufügt. Es ist immer ein schlechter gesundheitlicher Zustand seiner Protagonisten, der einen plausiblen thematischen Rahmen jeder Überschreitung von Normen bildet. Er wirkt wie eine Rechtfertigung aller Strategien, die diese Protagonisten im Dienste des Geschlechts, des Begehrens entwickeln, um mit einer weiteren Frau intim verkehren zu können. In diesem unspezifischen, fiebrigen $\mathrm{Zu}-$ stand kann den Agierenden, Träumenden, Erlebenden keine Verantwortung abverlangt werden. Sie selber leiden, wie oben gezeigt, genug. Sie fiebern, und dadurch passiert etwas, was jenseits von Gut und Böse ist, weil sie sich dagegen nicht wehren können, wie Erik Falk, der benommen und nicht imstande ist, die Frauen zu meiden, auch wenn er weiß, dass sie durch ihn unglücklich werden. Przybyszewski konstruiert Situationen, in denen seine Figuren in ein unlösbares Dilemma geraten: Wenn sie sich ihren Wünschen, Gelüsten, ihrem Begehren hingeben, verletzen sie alle, die sie lieben, und sie selbst leiden darunter. Wenn sie ihren innigen Wünschen nicht nachgehen, verschlechtert sich ihr psychischer Zustand, das Begehren intensiviert sich, raubt ihnen die Vernunft und die Hoffnung auf rationales Handeln. Ihre Temperatur steigt. Beim Begehren geht es bei Przybyszewski nicht um Untreue, sondern um eine unverständliche Unausweichlichkeit des sinnlichen Geschehens, das im Widerspruch zur konventionellen Liebesauffassung steht, die Falk in der Trilogie ablehnt. Die Ambivalenz, die Przybyszewski thematisiert, ergibt sich bezeichnenderweise nicht nur aus den Erwartungen der Geliebten, sondern auch aus der inneren Überzeugung der Liebenden, sonst würden sie nicht so leiden wie Falk. Diese Ambivalenz des Begehrens, die Przybyszewskis Figuren immer wieder erfahren und erleben, indem sie zwischen der Norm/Erwartung und Erfüllung taumeln, ist daher mehr als nur ein Teil seiner Schreibstrategie, um die Geschlechtsproblematik bei der Lektüre seiner Werke erträglicher zu machen. Es ist auch sein existenzielles Anliegen, dieser Aporie der menschlichen Existenz auf die Spur zu kommen, deren Quelle er in der Verdrängung des Geschlechts durch die Ratio sah. Für Przybyszewski war die Erkenntnis ausschlaggebend, wie gern seine Zuhörer und Zuhörerinnen Rauschzustände genossen. In seinem Erinnerungsbuch Ferne komm ich her... Erinnerungen an Berlin und Krakau von 1930 sucht er nach deren Gründen im Zusammenhang mit seiner Art, Werke Chopins zu spielen:

So manches Mal habe ich mir den Kopf zerbrochen, was es mit meinem durch und durch dilettantischen Spiel auf sich hatte, daß es die Leute so mitriß, pah! Sie völlig aus dem Gleichgewicht brachte. Mir scheint, es machte die für gewöhnlich stark erregte Stimmung, die den Zuhörern verwehrte, 
die fatalen technischen Mängel meines zügellosen Spiels kritisch zu betrachten. Ihr Gehör ward taub für die virtuose Seite des Vortrags, um so empfänglicher ward die Seele für den Geist des Werkes, den ich mit meinem barbarischen Spiel entfesselte. ${ }^{42}$

Dieses Klavierspiel mit solch einer Bravour und Zügellosigkeit versetzte Przybyszewskis Zuhörer und Zuhörerinnen in eine Art Ekstase, einen begehrenden $\mathrm{Zu}$ stand, den sie immer wieder erleben wollten, von dem sie den anderen erzählten und so für eine Vergrößerung seines Publikums sorgten. Auf diese Weise ist unter anderem Richard Dehmel zu solch einem „Konzert“ gekommen. Er formulierte seinen Wunsch direkt: „Heute wirst du spielen, nur für mich wirst Du spielen! ${ }^{‘ 33}$ So begann eine tiefe Freundschaft zwischen ihm und Przybyszewski, der genau wusste, dass sein Spiel „zum stärksten Bindeglied zwischen“ ihm und seinen „deutschen Schriftstellerbrüdern“ wurde. ${ }^{44}$ Diese Übertreibung bis zur Verausgabung wurde in Przybyszewskis Umgebung Chopenisierung genannt und hatte einen großen Einfluss nicht nur auf Richard Dehmel, sondern auch auf Julius Hart, Johannes Schlaf u. a. Przybyszewski war in deren Umgebung ein Fremder, der sich mehr erlauben durfte als die einheimischen Schriftsteller. Er konnte Normen jeglicher Art überschreiten, womit er die anderen beeindruckte, die es gerne auch gemacht hätten, aber es noch nicht wagten, wie eben Richard Dehmel. Es sei nur an eines seiner Gedichte erinnert, in dem er den Widerspruch zwischen der ethischen Haltung und dem Geschlecht im Sinne Przybyszewskis anspricht:

Der gesunde Mann

Meine Frau ist krank, sie

wird wohl bald sterben;

dann kann ich lachen,

dann werd' ich was erben.

O, wie lieb mir das Leben im Leibe schlägt, wenn ihr Husten mir das Herz zersägt,

o Gott!

Da sitzt sie am Ofen

und lächelt ins Feuer;

die Flammen röcheln

so ungeheuer.

Es kocht die Glut, ein Scheit zerspringt,

und eine ferne Glocke klingt.

O Gott! $!^{45}$

${ }^{42}$ Stanisław Przybyszewski: Ferne komm ich her... Erinnerungen an Berlin und Krakau. Aus dem Polnischen übertragen von Roswitha Matwin-Buschmann. Leipzig/Weimar 1985, S. 113.

${ }^{43}$ Ebd.

${ }^{44}$ Ebd., S. 114.

${ }^{45}$ Richard Dehmel: Der gesunde Mann, <http://www.bookrix.com/book.html?bookID=bx.dehmel_ 1298390798.1380550861\#522,522,1134> [12.12.2015], [Hervorhebung - B.C.]. 
Hier ist nicht nur der Erzählduktus ein anderer als bei Przybyszewski. Es geht auch um eine andere Krankheit. Während eine todkranke Ehefrau auf dem Sterbebett liegt, sehnt sich ihr Ehemann nach ihrem Tod, weil er nur dann sich ausleben kann. Aber der Mann empfindet keine kochende Glut, diese ist im Ofen, in dessen Flammen die Kranke und er schauen, für die sie aber je etwas anderes symbolisiert. Bei Przybyszewski fiebern männliche Protagonisten und folgen ihrem Begehren, ohne zu zögern. In polyphonen Formen seiner Prosa geht Przybyszewski das Thema der Ambivalenz des sexuellen Erlebnisses immer wieder neu an, indem er einerseits das Begehren aufzuwerten versucht und andererseits das permanente Scheitern beschreibt, zu dem jedes Individuum durch die Ratio, die gesellschaftlichen Normen und die Liebenden oder durch sich selbst verurteilt wird. Sein Publikum bleibt bei der Lektüre auf der sicheren Seite. Es wird befriedigt und beruhigt zugleich, denn Przybyszewski chopenisiert zwar, lässt aber alles im fiebrigen Zustand seiner Protagonisten geschehen. Auf diese Weise verpflichtet er keinen zur Stellungnahme, bietet aber ein besonderes Vergnügen beim Lesen seiner ,hochtemperierten“ Texte.

\section{Literatur}

Badowska, Katarzyna: Godzina cudu. Miłość i erotyzm w twórczości Stanisława Przybyszewskiego. Łódź 2011.

Fischer, Jens Malte: Fin de siècle. Kommentare zu einer Epoche. München 1978.

Matuszek, Gabriela: Między pustka transcendencji a szaleństwem zmystów. O wczesnej eseistyce Stanisława Przybyszewskiego. Kraków 1997.

Przybyszewski, Stanisław: Ferne komm ich her... Erinnerungen an Berlin und Krakau. Aus dem Polnischen übertragen von Roswitha Matwin-Buschmann. Leipzig/Weimar 1985.

Przybyszewski, Stanisław: Im Malstrom. In: ders.: Homo Sapiens. Bd. III. Paderborn 1993, S. 107-309.

Przybyszewski, Stanisław: Totenmesse. In: ders.: Werke, Aufzeichnungen, Briefe. Bd. I. Paderborn 1990, S. 7-41.

Przybyszewski, Stanisław: Über Bord. In: ders.: Homo Sapiens. Bd. I. Paderborn 1993, S. 13-109.

Przybyszewski, Stanisław: Unterwegs. In: ders.: Homo Sapiens. Bd. II. Paderborn 1993, S. 111-196.

Przybyszewski, Stanisław: Zur Psychologie des Individuums. I. Chopin und Nietzsche [online], $<$ https://wolnelektury.pl/katalog/lektura/przybyszewski-zur-psychologie-des-individuums.html>.

Steltner, Ulrich: Stanisław Przybyszewski - Schriftsteller in zwei Nationalliteraturen [online], <http://www.slawistik.uni-jena.de/tl_files/tl_files/Institutsverlag/Kollar/11steltner.pdf>. 